\title{
Adesão ao tratamento e patologias relacionadas em pacientes insulinodependentes no Município de Corbélia - Paraná
}

\author{
Adhesion to treatment and related pathologies in insulin dependent patients in the City of Corbélia \\ - Paraná
}

Adhesión al tratamiento y patologías afines en pacientes insulinosos del Municipio de Corbélia -

Paraná

Recebido: 12/10/2021 | Revisado: 19/10/2021 | Aceito: 20/10/2021 | Publicado: 23/10/2021

\author{
Antony Bontempo Garcia \\ ORCID: https://orcid.org/0000-0002-8350-0224 \\ Centro Universitário Fundação Assis Gurgacz, Brasil \\ E-mail: abgarcia@minha.fag.edu.br \\ Vagner Fagnani Linartevichi \\ ORICID: https://orcid.org/0000-0002-2624-7744 \\ Centro Universitário Fundação Assis Gurgacz, Brasil \\ E-mail linartevichi@fag.edu.br
}

\begin{abstract}
Resumo
Introdução: o paciente diabético muitas vezes não adere ao tratamento com insulina, mesmo com todas as adversidades que a patologia possa vir o proporcionar. Objetivo: realizar um levantamento em uma farmácia básica do oeste do Paraná, com intuído de analisar a adesão ao tratamento dos pacientes à insulina e possíveis patologias concomitantes que os mesmos possam apresentar. Metodologia: realizaram-se entrevistas através de um questionário semiestruturado onde os participantes foram pacientes que realizaram a retirada de insumos na farmácia. Resultados: entrevistou-se 50 pacientes onde foram 25 homens (50\%) e 25 mulheres (50\%), com idade média de 64 anos, o índice de massa corpórea (IMC) mais presente foi de sobrepeso (32\%), as patologias mais presentes em ambos os grupos foram hipertensão (84\%) homens e (88\%) mulheres e dislipidemia (64\%) para ambos, os medicamentos mais utilizados no grupo masculino foram Sinvastatina (56\%), Losartana $(36 \%)$ e para mulheres, Losartana $(56 \%)$ e Sinvastatina (48\%). Conclusão: As patologias mais presentes foram hipertensão e dislipidemia, porém não apresentou grande divergência entre os grupos, o tratamento mais preferível foi com losartana e sinvastatina.
\end{abstract}

Palavras-chave: Diabetes; Insulina; Tratamento.

\begin{abstract}
Introduction: the diabetic patient often does not adhere to insulin treatment, even with all the adversities that the pathology may provide. Objective: to carry out a survey in a basic pharmacy in western Paraná, with the aim of analyzing patients' adherence to insulin treatment and possible concomitant pathologies that they may present. Methodology: interviews were carried out through a semi-structured questionnaire where the participants were patients who carried out the withdrawal of supplies at the pharmacy. Results: 50 patients were interviewed where they were 25 men (50\%) and 25 women $(50 \%)$, with a mean age of 64 years, the most present body mass index (BMI) was overweight (32\%), the pathologies more present in both groups were hypertension $(84 \%)$ men and (88\%) women and dyslipidemia (64\%) for both, the most used drugs in the male group were Simvastatin (56\%), Losartan (36\%) and for women , Losartan (56\%) and Simvastatin (48\%). Conclusion: The most common pathologies were hypertension and dyslipidemia, but there was no great divergence between the groups, the most preferable treatment was with losartan and simvastatin.
\end{abstract}

Keywords: Diabetes; Insulin; Treatment.

\section{Resumen}

Introducción: el paciente diabético muchas veces no se adhiere al tratamiento insulínico, incluso con todas las adversidades que la patología puede aportar. Objetivo: realizar una encuesta en una farmacia básica del occidente de Paraná, con el objetivo de analizar la adherencia de los pacientes al tratamiento con insulina y las posibles patologías concomitantes que pueden presentar. Metodología: las entrevistas se realizaron mediante un cuestionario semiestructurado donde los participantes fueron pacientes que realizaron la retirada de insumos en la farmacia. Resultados: se entrevistaron 50 pacientes donde fueron 25 hombres $(50 \%)$ y 25 mujeres (50\%), con una edad media de 64 años, el índice de masa corporal (IMC) más presente fue el sobrepeso (32\%), las patologías más presentes en ambos grupos fueron hipertensión (84\%) hombres y (88\%) mujeres y dislipidemia (64\%) para ambos, los fármacos más 
utilizados en el grupo masculino fueron Simvastatina (56\%), Losartán (36\%) y para mujeres, Losartán (56\%) y Simvastatina (48\%). Conclusión: Las patologías más frecuentes fueron hipertensión y dislipidemia, pero no hubo gran divergencia entre los grupos, el tratamiento más preferible fue con losartán y simvastatina.

Palabras clave: Diabetes; Insulina; Tratamiento.

\section{Introdução}

Nos dias atuais o diabetes mellitos tipo 2 (DM2) se caracteriza por ser uma patologia que atinge em sua maioria pacientes acima dos 30 anos com histórico familiar relacionado à doença, porém a taxa de pacientes vem aumentando devido à industrialização (Macedo et al., 2019). O consumo de produtos com altos teores de açúcares, inatividade física, sobre peso e alguns transtornos que levam o paciente comer exageradamente, a demora no diagnóstico é um problema em grande parte dos casos tardando o início do tratamento, pois os sintomas não são muito frequentes no início, o pouco acompanhamento profissional que ocorre após o diagnóstico dificulta a adesão do paciente ao tratamento, piorando o quadro sendo necessário tratamentos mais rigorosos e intensivos, estes diversos fatores tem influenciado muito na taxa de pacientes que vem desenvolvendo tal patologia (Maraschin et al., 2010).

A insulinoterapia em pacientes com DM2 é administrada, de acordo com o grau de hiperglicemia que deve ser automonitorada diariamente com o auxílio de um glicosímetro e sua tira própria que utiliza uma pequena quantidade de sangue normalmente retirada dos capilares da ponta dos dedos, o uso da insulina pode ser concomitante com antidiabéticos orais ou não (Melo, 2006; Oliveira et al., 2020).

De acordo com a sociedade brasileira de diabetes em um estudo realizado pela International Diabetes Federation (IDF) no ano de 2017 consta que o Brasil apresentava cerca de 12,5 milhões de casos de DM aparecendo em $4^{\circ}$ lugar no ranking mundial atrás de Índia, China e Estados Unidos, países com maior população, em uma pesquisa realizada com pacientes entre 20 e 79 anos, cerca de $79 \%$ dos casos vivem em países em desenvolvimento, nos quais o aumento será maior nas próximas décadas segundo algumas estimativas (Minella \& Linartevichi, 2021; SBD, 2019).

Pacientes que possuem a DM2 em sua maioria são de idade mais avançada, sendo a maior parte em idosos pouco alfabetizados ou analfabetos, o que dificulta a adesão ao tratamento, o pouco acompanhamento profissional adequado também é um fator gritante e tende a ser melhorado principalmente na rede de saúde pública, segundo (OMS) estima que a glicemia elevada seja o terceiro fator que mais causa mortalidade prematura, atrás apenas do tabaco e a pressão arterial, coisa que não vem sendo levado muito em consideração pelo sistema de saúde pública (SBD, 2019).

Dado este exposto, o presente trabalho teve por objeto avaliar o índice de comorbidades em pacientes com DM2 no município de Corbélia-Pr, assim como seu grau de adesão ao tratamento.

\section{Metodologia}

Estudo foi desenvolvido em duas etapas, a primeira seccional do tipo transversal (conforme descrito por Pereira et al., 2018), realizada na Farmácia Básica Municipal de Corbélia, estado do Paraná, e a segunda uma análise estatística dos dados coletados onde foram dispostos em uma planilha do software Microsoft Office Excel $2007 囚$.

$\mathrm{Na}$ primeira etapa foi realizada a aplicação de um questionário semiestruturado para entrevista com 50 pacientes diabéticos e insulinodependentes, usuários da farmácia Básica, onde recebiam os medicamentos para diversas patologias, insulinas e insumos para realização do automonitoramento do DM2. Não houve um planejamento amostral e a amostra foi obtida por conveniência, sendo entrevistados pacientes que foram até a farmácia para retirada dos insumos nos dias em que a pesquisa foi realizada desde que o mesmo se encaixa dentro dos critérios exigidos para a realização da pesquisa e concordar com os termos assinando o termo de consentimento livre e esclarecido (TCLE). 
Para a coleta de dados utilizou-se um instrumento de coleta semiestruturado em cinco módulos: o primeiro de dados sociodemográficos, composto das seguintes variáveis: sexo, idade, cor/raça, estado civil, religião, renda familiar, coabitação. O segundo módulo foi composto de variáveis que visavam avaliar situações de risco como hábito de beber e fumar, sobrepeso e obesidade, atividade física e dietas. O terceiro módulo avaliou a relação do paciente com a DM2 e sua automonitorização: o tempo de diagnostico para DM2, se sente algum sintoma constante relacionado ao DM2, quantas vezes ao dia realiza a automonitoramento da glicemia capilar, a quanto tempo, se sente dificuldade, e os resultados da última semana. O quarto módulo consistiu em analisar a relação do paciente outras comorbidades e os tratamentos em uso: se tem diagnostico para outras doenças e quais, quando foi esse diagnostico, se usa outros medicamentos e quais, se sabe para quais patologias os medicamentos são utilizados. O quinto e último módulo teve como objetico avaliar a relação dos pacientes com a aplicação da insulina, qual insulina utiliza, quantas vezes ao dia, a quanto tempo, quantas unidades ao dia, se já esqueceu de utilizar, com que frequência, se já sentiu reações adversas ao aplicar, e se deixar de aplicar por isso, se tem dificuldade para aplicar, quantas vezes utiliza a mesma seringa e como são descartadas e armazenadas.

$\mathrm{Na}$ segunda etapa ocorreu que os dados coletados foram dispostos em uma planilha no software Microsoft Office Excel 2007, onde foram analisados paciente a paciente sem a utilização de software estatístico especifico, os resultados foram expressos de modo descritivo e em tabelas. A normas éticas foram obedecidas, o projeto do estudo foi submetido a apreciação de um Comitê de Ética em Pesquisa Envolvendo Seres Humanos da FAG - Centro universitário da Fundação Assis Gurgacz, e foi aprovado conforme o Parecer de Número: 4.779.440, CAAE 4630021 6.0000.5219.

\section{Resultados e Discussão}

A amostra foi constituída por 50 pacientes com DM2 que realizavam uso de insulina, onde foram entrevistados 25 homens (50\%) e 25 mulheres (50\%), a idade média dos entrevistados foi de 64 anos, variando de 37 a 88 anos. Em relação ao índice de massa corpórea (IMC) (32\%) dos entrevistados apresentaram sobrepeso, em relação ao estado civil (50\%) apresentaram-se casados, a maior parte eram da etnia branca (62\%), em relação a renda a maioria relatou ganhar entre 1.100 e $1.300 \mathrm{R}$ \$ por mês (54\%). A religião predominante foi a católica (90\%), (84\%) relatou não morar sozinho, dados presentes na (Tabela 1). Em um estudo realizado no município de Marialva - PR, foram apresentados dados sociodemográficos muito semelhantes ao do estudo atual, no estudo entrevistaram-se 30 pacientes diabéticos insulinodependentes que foram atendidos na unidade básica de saúde, em sua maioria foram pacientes do sexo feminino $(66,7 \%)$, com cor/etnia branca ( $70 \%)$, casados $(53,3 \%)$, com renda familiar de até dois salários-mínimos $(46,7 \%)$ e apenas $6,7 \%$ dos pacientes relatou morar sozinho (Soares \& Romanichen, 2020).

Com relação aos hábitos de vida dos pacientes entrevistados (18\%) relataram fumar diariamente, já a quantidade de paciente que relataram ter fumado no passado foi de (48\%), diversos motivos foram apresentados pelos pacientes como motivo para ter parado de fumar, entre eles o mais frequente foi que percebeu que não fazia bem (6\%), os entrevistados relataram ter parado de fumar a mais de 10 anos e (18\%). Em questão ao consumo de bebidas alcoólicas, (14\%) relatou realizar uso ocasionalmente, quando perguntados se já fizeram uso de bebidas alcoólicas no passado o percentual subiu razoavelmente para $(50 \%)$ dos entrevistados, o motivo mais comum que levou os pacientes a para o consumo de álcool para o grupo foi que "parou junto com o cigarro" (14\%). Grande parte parou de beber a mais de 10 anos (22\%). 
Tabela 1. Características sociodemográficas de usuários de insulina do sistema público de saúde no município Corbélia - PR.

\begin{tabular}{|c|c|c|}
\hline & $\mathbf{N}$ & $\%$ \\
\hline \multicolumn{3}{|l|}{ Sexo } \\
\hline Homens & 25 & $50 \%$ \\
\hline Mulheres & 25 & $50 \%$ \\
\hline \multicolumn{3}{|l|}{ IMC } \\
\hline Sobrepeso & 16 & $32 \%$ \\
\hline Obesidade I & 15 & $30 \%$ \\
\hline Obesidade II & 7 & $14 \%$ \\
\hline Obesidade III & 2 & $4 \%$ \\
\hline Normal & 10 & $20 \%$ \\
\hline \multicolumn{3}{|l|}{ Estado civil } \\
\hline Casado & 25 & $50 \%$ \\
\hline Viúvo & 15 & $30 \%$ \\
\hline Solteiro & 6 & $12 \%$ \\
\hline Separado & 3 & $6 \%$ \\
\hline União estável & 1 & $2 \%$ \\
\hline \multicolumn{3}{|l|}{ Etnia } \\
\hline Branco & 31 & $62 \%$ \\
\hline Pardo & 12 & $24 \%$ \\
\hline Negro & 7 & $14 \%$ \\
\hline \multicolumn{3}{|l|}{ Renda ( R\$ ) } \\
\hline Menos de um salário mínimo & 3 & $6 \%$ \\
\hline $1.100-1.300$ & 27 & $54 \%$ \\
\hline $1.310-1.860$ & 4 & $8 \%$ \\
\hline $1.870-2.488$ & 10 & $20 \%$ \\
\hline $2.489-3.110$ & 3 & $6 \%$ \\
\hline Acima de 3.111 & 3 & $6 \%$ \\
\hline \multicolumn{3}{|l|}{ Religião } \\
\hline Católica & 45 & $90 \%$ \\
\hline Evangélica & 5 & $10 \%$ \\
\hline \multicolumn{3}{|l|}{ Reside sozinho } \\
\hline Sim & 8 & $16 \%$ \\
\hline Não & 42 & $84 \%$ \\
\hline
\end{tabular}

Comparação em percentual dos dados sociodemográficos de pacientes masculinos e femininos insulinodependentes no município de Corbélia - PR. Fonte: Autores.

Entre o grupo de entrevistados apresentou (44\%) pratica de exercícios físicos, onde todos relataram a pratica de caminhada a principal causa relatada da não prática de exercícios foi à dificuldade em realizar as atividades (44\%). Os hábitos alimentares se demonstraram uma dificuldade onde (68\%) disseram não realizar alguma dieta indicada por um profissional, para os que deixaram de comer algo o alimento mais retirado da alimentação em foi o pão branco (24\%), o principal fato não vê necessidade de deixar de comer algumas coisas (28\%) presentes na (Tabela 2). No estudo realizado no município de Tubarão-SC, foram entrevistados 40 pacientes, onde 2 não se encaixaram nos critérios para participar da pesquisa, o estudo apresentou dados que corroboram aos do presente estudo, maior parte dos entrevistados se apresentou inativo (63,2\%) e para os que apresentaram ser ativos a caminhada de rua de uma a duas vezes na semana foi o exercício mais praticado, a maioria relatou ter mudado a alimentação após o diagnóstico da DM2, (7,8\%) dos pacientes eram tabagismo ativos, mas em relação a o ex-tabagismo o número aumentou razoavelmente para (60,5\%) (Schuelter et al., 2019). No estudo de Marialva-PR, também demonstrou resultados semelhantes em relação a tabagismo e o consumo de bebidas alcoólicas onde (6,7\%) relataram fumar, porém para o consumo de bebidas alcoólicas o número subiu para (26,7\%) (Soares \& Romanichen, 2020). 
Tabela 2. Hábitos de usuários de insulina do sistema público de saúde no município Corbélia - PR.

\begin{tabular}{|c|c|c|}
\hline & $\mathbf{N}$ & $\%$ \\
\hline \multicolumn{3}{|l|}{ Fuma } \\
\hline Sim, diariamente & 9 & $18 \%$ \\
\hline Não & 41 & $82 \%$ \\
\hline \multicolumn{3}{|l|}{ Já fumou } \\
\hline Sim & 24 & $48 \%$ \\
\hline Não & 26 & $52 \%$ \\
\hline \multicolumn{3}{|l|}{ Porque parou } \\
\hline Nunca fumou & 26 & $52 \%$ \\
\hline Não parou & 9 & $18 \%$ \\
\hline Percebeu que não fazia bem & 3 & $6 \%$ \\
\hline Por causa dos medicamentos & 2 & $4 \%$ \\
\hline Outros & 10 & $20 \%$ \\
\hline \multicolumn{3}{|l|}{ Faz uso de álcool } \\
\hline Sim, diariamente & 1 & $2 \%$ \\
\hline Sim, ocasionalmente & 7 & $14 \%$ \\
\hline Não & 42 & $84 \%$ \\
\hline \multicolumn{3}{|l|}{ Já fez uso de álcool } \\
\hline Sim & 25 & $50 \%$ \\
\hline Não & 25 & $50 \%$ \\
\hline \multicolumn{3}{|l|}{ Porque parou } \\
\hline Não parou & 8 & $16 \%$ \\
\hline Nunca bebeu & 25 & $50 \%$ \\
\hline Parou junto com o cigarro & 7 & $14 \%$ \\
\hline Outros & 10 & $20 \%$ \\
\hline \multicolumn{3}{|l|}{ Pratica exercícios físicos } \\
\hline $\operatorname{Sim}$ & 22 & $44 \%$ \\
\hline Não & 28 & $56 \%$ \\
\hline \multicolumn{3}{|l|}{ O que pratica } \\
\hline Nada & 28 & $56 \%$ \\
\hline Caminhada & 22 & $44 \%$ \\
\hline Exercícios no parque & 2 & $4 \%$ \\
\hline
\end{tabular}

Comparação em percentual de hábitos diários dos pacientes masculinos e femininos insulinodependentes no município de Corbélia - PR. Fonte: Autores.

Grande parte dos pacientes relatou que os sintomas do DM2 melhoram após iniciar o tratamento com a insulina (100\%) homens e $(96 \%)$ das mulheres, também relataram melhora no HGT (92\%) homens e (76\%) das mulheres, para o exame de hemoglobina glicada (HbA1c) os pacientes também relataram melhora em maior parte dos casos para o grupo masculino (56\%), (28\%) dos pacientes não lembraram ou não tinham os resultados, no caso do grupo feminino (44\%) dos pacientes apresentou melhora nos resultados, apenas (12\%) não lembrou ou não possuía os resultados, o tempo de diagnóstico para DM2 do grupo masculino variou de 2 anos a 32 anos, com (44\%) dos pacientes tendo diagnóstico entre 2 anos e 10 anos, e (56\%) 11 anos ou mais, para as mulheres a variação foi de 2 anos a 30 anos, tendo (60\%) dos pacientes com diagnóstico entre 2 e 10 anos, e (40\%) 11 anos ou mais.

A maioria dos pacientes disseram fazer o teste de glicemia capilar diariamente, $(88 \%)$ dos homens relataram realizar os testes, os resultados mais frequentes relatados pelos pacientes foram entre $100 \mathrm{mg} / \mathrm{dL}$ e $150 \mathrm{mg} / \mathrm{dL}$ (56\%), no caso do grupo feminino $(72 \%)$ das pacientes relataram realizar os testes diariamente e os resultados mais frequentes foram entre $100 \mathrm{mg} / \mathrm{dL}$ e $150 \mathrm{mg} / \mathrm{dL}(40 \%)$ dos casos. A dificuldade na realização dos testes esteve presente em (40\%) das mulheres entrevistadas e (12\%) dos homens, (Tabela 3).

Em um estudo o autor ressaltou o monitoramento de glicose contínuo ele relata a importância desses testes para pacientes com DM2, o que traz maior facilidade ao tratamento e melhor adequação quando necessário, o estudo também 
salientou o exame de $\mathrm{Hb}$ A1c e sua importância para a população principalmente para o grupo em risco de complicações vasculares (Calson, Mullen \& Bergental, 2018).

Tabela 3. Relação dos pacientes com DM2 e automonitorização da glicemia capilar de usuários de insulina do sistema público de saúde no município Corbélia - PR.

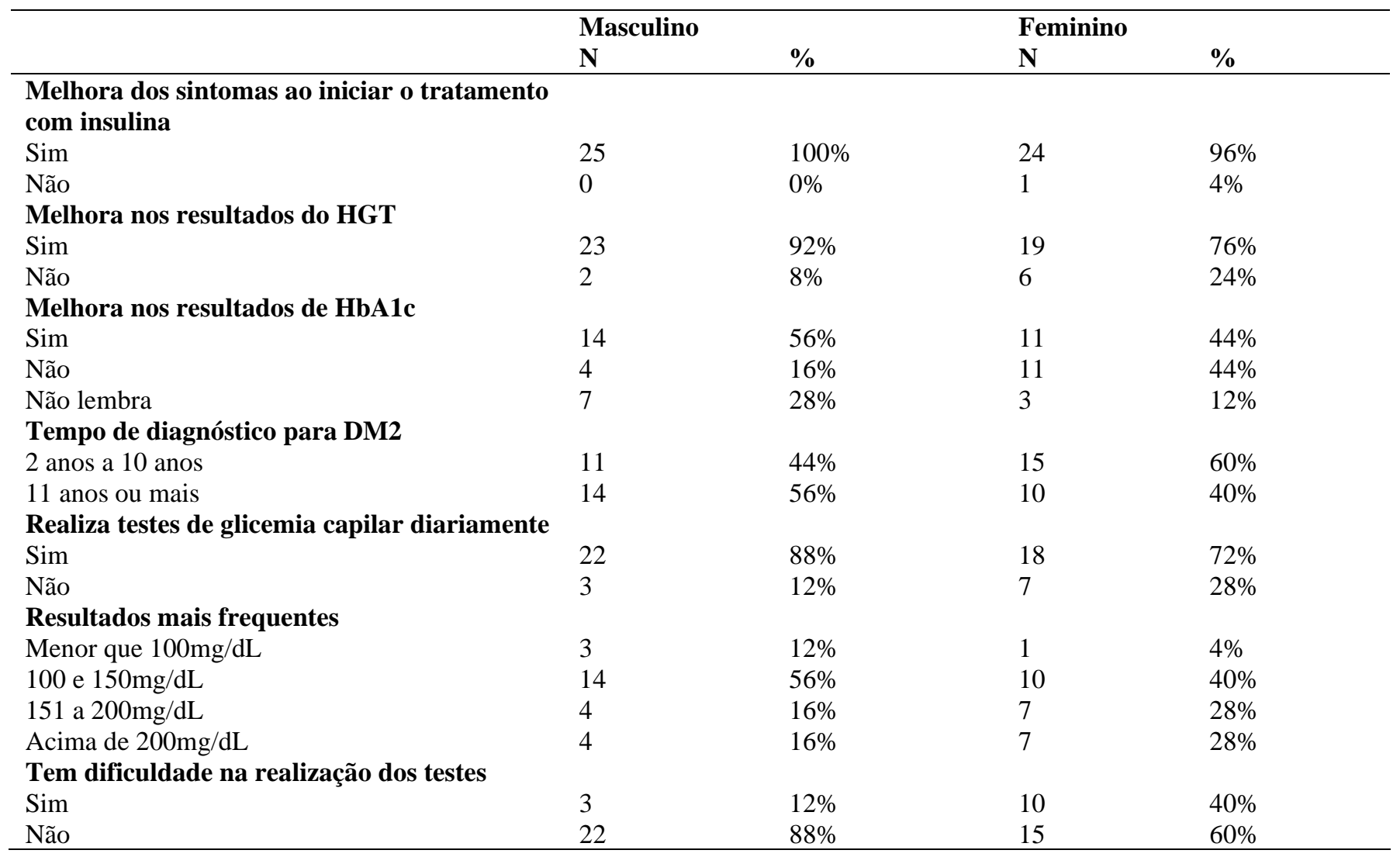

Comparação em percentual da relação dos pacientes insulinodependentes do sexo feminino e masculino com o DM2 e seu monitoramento diário no município de Corbélia - PR. Fonte: Autors.

Em um estudo de cuidado farmacêutico onde houve acompanhamento de um grupo foi obtido resultados muito favoráveis onde uma paciente obteve $40 \%$ de redução da glicemia de jejum, $67,77 \%$ na pós-prandial e 22,73\% na HbA.1c, onde ele também relatou haver carência nas orientações ao paciente sobre o armazenamento e uso correto dos hipoglicemiantes sedo assim necessário de melhor preparo das equipes para obtenção de melhores resultados nas terapias (Oliveira et al., 2019).

De acordo com outro estudo apesar da introdução de uma insulina basal, mais de 70\% dos pacientes não alcançam um bom controle glicêmico devido ao medo de hipoglicemia e ganho de peso, falta de motivação e a incapacidade de titular a dose de forma adequada são os principais obstáculos para atingir os objetivos do tratamento e seu manejo é parte integrante do processo de tratamento, destaca também a grande necessidade de maior atenção à educação terapêutica sendo essencial para a melhor adesão ao tratamento (Giorgino, Molfetta \& Caruso, 2020; Schonrock et al., 2021).

Com relação às demais patologias, 24 homens (96\%) apresentaram ter outras patologias além do DM2 e 25 mulheres (100\%) apresentaram o mesmo, a hipertensão foi à patologia mais presente em ambos os grupos $(84 \%)$ homens e $(88 \%)$ mulheres, logo a frente da dislipidemia acometendo (64\%) dos homens e mulheres, a ansiedade/depressão e insônia se demonstraram presentes em um número maior de mulheres em relação aos homens, (24\%) homens e (44\%) mulheres, o grupo masculino apresentou ligeiramente maior número de pacientes com hipotiroidismo (16\%) e (12\%) mulheres. As mulheres de modo geral apresentaram ter mais patologias concomitantes que os homens, (48\%) das mulheres apresentaram ter 5 patologias 
ou mais, enquanto, para homens foram (20\%) dos pacientes com 5 patologias ou mais, ambos grupos a maioria dos entrevistados relataram que as demais patologias foram diagnosticadas após o DM2. De modo geral foram apresentadas 13 patologias diferentes pelo grupo masculino e 16 patologias diferentes pelo grupo feminino. Os medicamentos mais utilizados no grupo masculino foram Sinvastatina (56\%), Losartana e AAS (36\%), Anlodipino, Enalapril e Hidroclorotiazida (28\%), enquanto para as mulheres, Losartana (56\%), Sinvastatina (48\%), AAS (36\%), Hidroclorotiazida (32\%) e Omeprazol (28\%), foram os mais utilizados. Entre os 25 homens entrevistados foram relatados o consumo de 33 medicamentos diferentes, apenas um paciente não lembrou o nome dos medicamentos que fazia uso, (64\%) dos homens relataram que consumiam 5 ou mais medicamentos diferentes ao dia, sem levar em consideração medicamentos para DM2, entre as 25 mulheres entrevistadas foram relatados o uso de 46 medicamentos diferentes, (64\%) relataram que consumiam 5 ou mais medicamentos diferentes ao dia (Tabela 4).

Tabela 4. Medicamentos mais utilizados e patologias relacionadas ao DM2 em usuários de insulina do sistema público de saúde no município Corbélia - PR

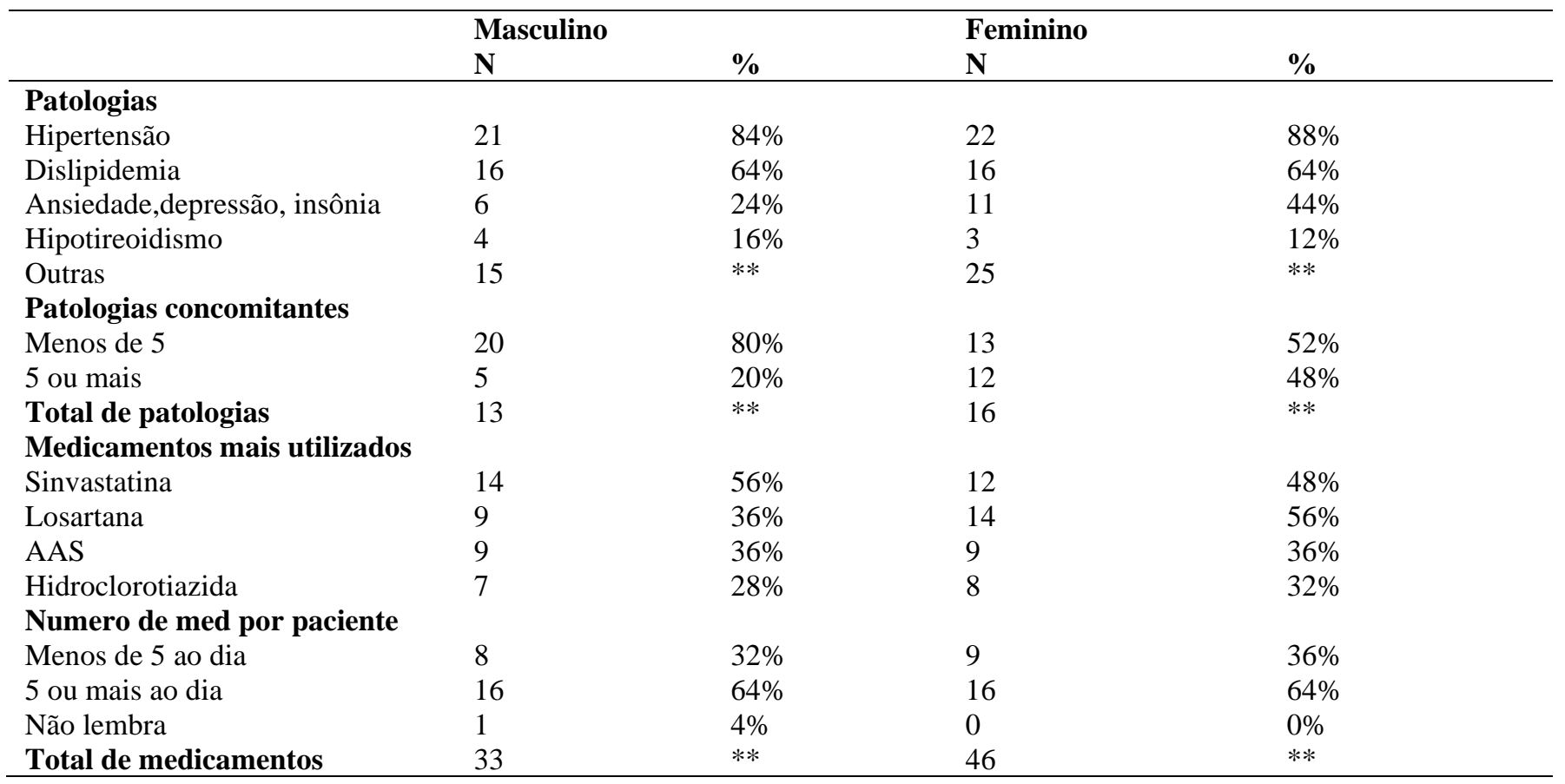

Comparação em percentual dos pacientes insulinodependentes do sexo feminino e masculino com o DM2, patologias relacionadas aos pacientes e os medicamentos mais utilizados pelos mesmos no município de Corbélia - PR. Fonte: Autores.

Ainda no estudo de Marialva-PR os pacientes apresentaram comorbidades concomitantes a DM2 muito semelhantes e que corroboram a do presente estudo, 50\% dos pacientes apresentaram HAS, 10\% sofriam de alguma doença neurológica, $20 \%$ doenças renais e 26,7\% algum transtorno gastrointestinal (Soares \& Romanichen, 2020). No estudo de Tubarão-SC as comorbidades concomitantes a DM2 apresentaram-se também muito semelhantes, com grande parte dos pacientes apresentando HAS 65,8\%, dislipidemia 31,6\%, doenças da tireoide, depressão ou ansiedade 13,2\% (Brito et al., 2020; Schuelter et al., 2019).

Para o tratamento de hipertenção os dados obtidos corroboram a os indicados pela SBD, onde o indicado para o tratamento de HAS quando o paciente possui o DM2 são medicamentos demonstradas para reduzir eventos cardiovasculares como inibidores da enzima conversora de angiotensina (IECA), bloqueadores do receptor de angiotensina (BRA), diuréticos tiazídicos ou bloqueadores dos canais de cálcio (SBD, 2020) 
$\mathrm{O}$ recomendado para pacientes que possuem o DM2 concomitante com as dislipidemias ainda é a atividade física acompanhado de dietas, para o tratamento farmacológico adequado os medicamentos que apresentam melhor eficácia são os da classe das estatinas que por sua vez detém mais evidencias sobre seus benefícios cardiovasculares, é importante levar consideração não apenas a redução da mortalidade por problemas cardiovasculares, mas também os riscos de pancreatite aguda (Maria et al., 2021).

Com relação a depressão um estudo de revisão realizado no ano de 2020 apresentou forte relação entre o DM2 e a depressão, relatando que a pessoa pode apresentar sintomas depressivos devido ao impacto social que a DM2 pode causar a vida do paciente, apontando que existe uma hipótese de que a depressão pode estar ligada diretamente com a resistência de insulina, causando o seu aumento, e com relação aos fatores fisiopatológicos do diabetes, os sintomas depressivos podem piorar na adesão do tratamento da doença, os medicamentos que demonstraram maior eficácia são os antidepressivos da classe dos ISRS (inibidores seletivos da recaptação da serotonina), apresentam um efeito positivo no tratamento da depressão em pacientes portadores do DM2. Os antidepressivos dessa classe mais estudados foram a Sertralina e a Fluoxetina e os mesmos foram eficazes no tratamento da depressão e uma melhora do controle glicêmico. (Rodrigues, Brito \& Alvim, 2020).

Muitos pacientes além de utilizar a insulina para controle glicêmico utilizam alguns hipoglicemiantes orais, como Metformina, Glibenclamida, Glimepirida, Gliclazida e Alogliptina+Metformina, o medicamento mais utilizado para o tratamento foi a metformina $850 \mathrm{mg}$ um comprimido três vezes ao dia $(26 \%)$, a segunda forma de tratamento mais utilizada foi o uso da insulina apenas (18\%), a insulina NPH foi utilizada por todos pacientes, já a regular apenas por (28\%) dos entrevistados, a variação de Ui aplicadas ao dia foi de 10Ui a 100Ui com (46\%) dos pacientes aplicando 50Ui ou mais e (54\%) aplicando 49Ui ou menos. Grande parte dos entrevistados relataram realizar rodízio e aplicar somente na região abdominal (52\%), o motivo mais frequente para realização de rodízio na aplicação foi o fato de ficar roxo o local se aplicar várias vezes (34\%), reações adversas foram outro ponto frequente, que foram apresentadas em (32\%) dos pacientes, a reação adversa mais frequente foi de ter baixado demais a glicemia, mas apareceram apenas no início do tratamento.

A dificuldade na aplicação esteve presente em (28\%) dos entrevistados e necessitavam de auxílio para a aplicação correta, outro fato presente foi à dificuldade na visão o que tornava difícil o preenchimento na seringa com as unidades corretas, (46\%) dos pacientes disseram já ter deixado de aplicar a insulina os principais motivos foram que quando se sentiam bem não aplicava e quando não estavam em casa principalmente no horário do almoço (Corgozinho et al., 2020). Com relação ao descarte dos materiais utilizados para aplicação da insulina e os testes de HGT, (78\%) dos descartar no coletor de perfuro, já o armazenamento das insulinas, todos relataram deixar na geladeira (tabela 5).

Em relação ao tratamento para a DM2 um estudo da associação coreana de diabetes demonstrou fatos que corroboram aos tratamentos utilizados por grande parte dos pacientes do estudo, a associação relatou que a terapia com insulina apresenta grande vantagem para melhorar o controle glicêmico quando comparado a medicamentos orais, porém apresenta riscos como podendo levar a ganho de peso e hipoglicemia em alguns casos, por isso ela por si só não é recomendada e sim sua associação com medicamentos orais, a indicação para uso de insulina logo no início do tratamento foi para pacientes com $\mathrm{Hb}$ A1c acima de 9\% onde a mesma apresenta redução nesses valores e também indicada em caso de insuficiência renal ou hepática, infarto agudo do miocárdio e acidente vascular cerebral (Lee et al., 2017). O estudo de Tubarão-SC também apresentou que $81,5 \%$ dos pacientes faziam em seu tratamento para DM2 o uso concomitante de insulina e hipoglicemiantes orais, onde em sua maioria o uso mais comum foi de Metfomina, logo seguido por Metformina e Glibenclamida (Schuelter et al., 2019). 
Tabela 5. Tratamentos farmacológicos para DM2 utilizados por usuários de insulina do sistema público de saúde no município Corbélia - PR.

\begin{tabular}{|c|c|c|}
\hline & $\mathbf{N}$ & $\%$ \\
\hline \multicolumn{3}{|l|}{ Tratamento hipoglicemiantes } \\
\hline Metformina $850 \mathrm{mg} 3$ comprimidos ao dia & 13 & $26 \%$ \\
\hline Insulina & 9 & $18 \%$ \\
\hline Metformina 500mg 3 comprimidos ao dia & 3 & $6 \%$ \\
\hline Metformina 500mg 4 comprimidos ao dia & 4 & $8 \%$ \\
\hline Outros & 21 & $42 \%$ \\
\hline \multicolumn{3}{|l|}{ Insulinas utilizadas } \\
\hline Somente NPH & 36 & $72 \%$ \\
\hline NPH e regular & 14 & $28 \%$ \\
\hline \multicolumn{3}{|l|}{ Unidades aplicadas ao dia } \\
\hline 50Ui ou mais & 23 & $46 \%$ \\
\hline 49Ui ou menos & 27 & $54 \%$ \\
\hline \multicolumn{3}{|l|}{ Onde aplicam a insulina } \\
\hline Barriga & 26 & $52 \%$ \\
\hline Barriga e coxa & 12 & $24 \%$ \\
\hline Barriga, braço e coxa & 5 & $10 \%$ \\
\hline Outros & 7 & $14 \%$ \\
\hline \multicolumn{3}{|l|}{ Realiza rodízio } \\
\hline Sim & 47 & $94 \%$ \\
\hline Não & 3 & $6 \%$ \\
\hline \multicolumn{3}{|l|}{ Porque realiza rodízio } \\
\hline Fica roxo se aplicar no mesmo local & 17 & $34 \%$ \\
\hline Para evitar aplicar sempre no mesmo local & 12 & $24 \%$ \\
\hline Dói se aplicar diversas vezes no mesmo local & 10 & $20 \%$ \\
\hline O médico orientou & 7 & $14 \%$ \\
\hline Tem medo de ficar cicatriz & 1 & $2 \%$ \\
\hline Não realiza & 3 & $6 \%$ \\
\hline \multicolumn{3}{|l|}{ Já deixou de aplicar a insulina } \\
\hline Sim & 23 & $46 \%$ \\
\hline Não & 27 & $54 \%$ \\
\hline \multicolumn{3}{|c|}{ Já apresentou reação adversa devido ao uso de insulina } \\
\hline Sim & 16 & $32 \%$ \\
\hline Não & 34 & $68 \%$ \\
\hline \multicolumn{3}{|l|}{ Tem dificuldade na aplicação da insulina } \\
\hline Sim & 14 & $28 \%$ \\
\hline Não & 36 & $72 \%$ \\
\hline \multicolumn{3}{|l|}{ Onde descarta os materiais utilizados } \\
\hline Coletor de perfuro & 39 & $78 \%$ \\
\hline Litro descartável & 9 & $18 \%$ \\
\hline Lixo separado & 2 & $4 \%$ \\
\hline \multicolumn{3}{|l|}{ Onde guarda a insulina } \\
\hline Geladeira & 50 & $100 \%$ \\
\hline
\end{tabular}

Demonstração em percentual dos pacientes insulinodependentes, seu tratamento farmacológico e modo da aplicação de insulina no município de Corbélia - PR. Fonte: Autores.

No estudo realizado no México onde participaram 200 pacientes que faziam uso de insulina, atendidos no centro terciário na Cidade do México, o estudo apresentou dados que demonstram os principais fatores para a não adesão a terapia com insulina como: falta de planejamento das atividades diárias $(46,1 \%)$, medo de hipoglicemia (41\%), fatores econômicos $(15,4 \%)$ e número de aplicações de insulina (Cabrera et al., 2018). Fatos que corroboram a algumas falas dos pacientes entrevistados "não uso quando me sinto bem" e "as vezes não dá tempo quando não estou em casa, principalmente na hora do almoço". 
Em um estudo realizado no município de Foz do Iguaçu-PR, analisou-se o armazenamento das insulinas pelos pacientes, onde a pesquisa obteve resultados que os pacientes não armazenavam corretamente as insulinas e em grande parte dos casos os pacientes em questão eram de idade entre 50 e 80 anos (Koch et al., 2019).

\section{Considerações Finais}

O DM2 é um problema social que vem crescendo constantemente, a insulinoterapia é uma opção para o tratamento muito efetiva, porém grande parte dos pacientes não adere ao tratamento por ser de certo modo desconfortável, a realização de rodízio na aplicação é preferível como demonstrado por $96 \%$ dos entrevistados, para evitar lesões e dor, outro fator que veio a interferir na adesão a insulinoterapia foi a dificuldade na aplicação em momentos em que o paciente não se encontra em casa, principalmente no horário do almoço, outro ponto foi a dificuldade em aplicar as unidades corretas. O tratamento para o DM2 mais utilizado foi de insulina NPH e metformina, é um medicamento muito utilizado, devido ao seu baixo custo e fácil aquisição pelo programa "aqui tem farmácia popular".

Dois terços dos pacientes apresentaram sobrepeso e obesidade I, muito devido à baixa pratica de exercícios físicos e adesão a dietas recomendadas por profissionais adequados.

Com relação as patologias concomitantes, foram analisadas em grupos separados de homens e mulheres, porém não houve grande divergência entre as patologias apresentadas, as mais comuns na maioria dos pacientes foram hipertensão e dislipidemia e os medicamentos mais utilizados foram Sinvastatina e a Losartana, que também são medicamentos de fácil acesso. O número de medicamentos utilizados pela maior parte dos pacientes foi de mais de 5 diferentes ao dia, outro fator esse que pode ser associado a maior dificuldade na adesão para o tratamento.

Dos entrevistados $32 \%$ relataram já ter sofrido com reação de hipoglicemia ao início do tratamento, e alguns relatos como "não uso quando me sinto bem" também foram apresentados, porém foram relatados melhora nos resultados de glicemia capilar e de HbA1c.

Através disso conclui-se devido aos relatos uma boa adesão ao tratamento com a insulinoterapia, no entanto, haja vista as características sociodemográficas e o perfil de patologias concomitantes, supõe-se que a adesão não seja ótima e deve-se direcionar o usuário para necessidade de ações educativas e tratamentos não farmacológicos para melhores cuidados da saúde e melhores resultados. O farmacêutico tem um importante papel na orientação e em atividades educativas a fim de melhorar a adesão destes pacientes ao tratamento farmacológico e às medidas não farmacológicos, como redução de fatores de risco e melhoria da qualidade de vida destes usuários dos serviços pública de saúde.

Neste contexto, sugere-se que novos estudos que visem avaliar a adesão ao tratamento em pequenas cidades sejam de imensa valia, uma vez que, há a produção de resultados passíveis de comparação e fornece aos órgãos públicos subsidio para que medidas preventivas possam ser elaboradas.

\section{Referências}

ADA. American Diabetes Association. (2021). Classificação e diagnóstico de diabetes: Padrões de atendimento médico em diabetes. Diabetes Care. https://doi.org/10.2337/dc21-S002.

Aguiar, K. G. L., Villela, R. N. \& Bouskela, E. (2007). A microcirculação no diabetes: implicações nas complicações crônicas e tratamento da doença. Arquivo brasileiro de Endocrinologia e Metabologia, (51)2, 204-211. http://dx.doi.org/10.1590/S0004-27302007000200009.

Balletshofer, L. B., Rittig, K., Enderle, M. D., Volk, A., Maerker, E., Jacob, S., Matthaei, S., Rett, K., \& Haring, H. U. (2000). A disfunção endotelial é detectável em parentes de primeiro grau jovens normotensos de indivíduos com diabetes tipo 2 em associação com resistência à insulina. American Heart Association, 101(15), 1780-1784. https://doi.org/10.1161/01.CIR.101.15.1780.

Brito, A. N. M. de, Macedo, J. L., Carvalho, S. de L., Oliveira, J. V. F. de, Brito, F. N. M., Pinheiro, A. O., Costa, P. V. de C., \& Silva, D. J. S. (2020). Effectiveness of diet therapy for patients with diabetes mellitus. Research, Society and Development, 9(1), e114911728. https://doi.org/10.33448/rsdv9i1.1728 
Cabrera, J. B., Valdes, A. P., Palacios, R. J., Salinas, A. C. A. \& Mehta, R. (2018). A adesão à insulina no diabetes tipo 2 no México: comportamentos e barreiras. Journal of Diabetes Research, 18(1), 1-7. https://doi.org/10.1155/2018/3190849.

Carlson, A. L., Mullen, D. M. \& Bergenstal, R. M. (2017). Clinical Use of Continuous Glucose Monitoring in Adults with Type 2 Diabetes. Diabetes Technology \& Therapeutics, 19(2), 4-11. https://doi.org/10.1089/dia.2017.0024.

Conselho Nacional de Saúde (2012). Resolução Conselho Nacional de Saúde $N^{o} 466$, de 12 de dezembro de 2012. https://bvsms.saude.gov.br/bvs/saudelegis/cns/2013/res0466_12_12_2012.html.

Corgozinho, M. L. M. V., Lovato, A. de C., Martins, I. C. de F., Mota, A. P. L., \& Mendes, A. C. R. (2020). Education in diabetes and changes in linving habits. Research, Society and Development, 9(3), e175932566. https://doi.org/10.33448/rsd-v9i3.2566

Ferreira, A. V. \& Campos, B. M. S. (2014). Avanços farmacológicos no tratamento do diabetes tipo 2. Bazilian Journal of Surgery and Clinical Research. 8(3), 72-78. https://www.ufjf.br/nfbio/files/2016/06/Tratamento-farmacol\%c3\%b3gico-DMT2.pdf.

Giorgino, F., Molfetta, S. \& Caruso, I. (2020). Manejo inicial da terapia com insulina basal no paciente diabético, L'Endocrinologo. 21(1), 370-373. https://doi.org/10.1007/s40619-020-00780-6.

Grillo, F. F. M., \& Gorini, C. P. I. M. (2007). Caracterização de pessoas com Diabetes Mellitus Tipo 2, Revista Brasileira de Enfermagem. 60(1), 49-54. https://doi.org/10.1590/S0034-71672007000100009.

Koch, M., Marin, M. P., Trindade, O. A. \& Piva, R. (2019). Avaliação sobre o armazenamento da insulina em uma amostragem de usuários. Revista Uningá, 56(1), 17-25. http://revista.uninga.br/index.php/uninga/article/view/2050/1878.

Lee, B., Kim, J. H., Ko, S. H., Hur, K. Y., Kim, N. H.., Rhee, S. Y., Kim, H. J., Moon, M. K., Park, S. O. \& Choi, K. M. (2017). Insulin Therapy for Adult Patient with Type 2 Diabetes Mellitus: A Position Statement of the Korean Diabetes Association. Diabetes \& Metabolism Journal, 41(5), 367373. https://doi.org/10.4093/dmj.2017.41.5.367.

Macedo, J. L., Oliveira, A. S. da S. S., Pereira, I. C., Reis, E. R., \& Assunção, M. de J. S. M. (2019). Epidemiological profile of diabetes mellitus in northeastern Brazil. Research, Society and Development, 8(3), e2883826. https://doi.org/10.33448/rsd-v8i3.826

Maraschin, J. F., Murussi, N., Witter, V. \& Silveiro, S. P. (2010). Classificação do Diabete Melito. Arquivo Brasileiro de Cardiologia. 9(2), 40-47. https://doi.org/10.1590/S0066-782X2010001200025.

Maria, O. I., Fonseca, F. A. H., Faludi, A. A. \& Araujo, D. B. (2021). Manejo do risco cardiovascular: dislipidemia. Diretriz 2021: Sociedade Brasileira de Diabetes. https://diretriz.diabetes.org.br/manejo-do-risco-cardiovascular-dislipidemia/\#ftoc-estratificacao-de-risco-em-pessoas-com-diabetes.

Matos, G. C. M. \& Branchtein, L. O. (2006). Uso de antidiabéticos orais no paciente com diabetes mellitus e doença cardíaca. Revista da sociedade de cardiologia do Rio Grande do Sul. 15 (8), 1-4. http://sociedades.cardiol.br/sbc-rs/revista/2006/08/o_uso_de_antidiabeticos.pdf.

Melo, K. (2006). Como e quando usar insulina no paciente com diabetes mellitus tipo 2: o papel do clínico/cardiologista. Revista da sociedade de cardiologia do Rio Grande do Sul.15(8), 1-6. http://sociedades.cardiol.br/sbc-rs/revista/2006/08/Como_e_quando_usar_insulina.pdf.

Minella, F. C. O., \& Linartevichi, V. F. (2021). Effects of cannabidiol on the signs and comorbidities of autistic spectrum disorder. Research, Society and Development, 10(10), e64101018607. https://doi.org/10.33448/rsd-v10i10.18607

Oliveira, L. C., Pires, G. B., Alencar, B. R., \& Alencar, T. O. S. (2019). Cuidados farmacêuticos em pessoas com diabetes em uso de insulina: relato de experiência. Revista de Divulgação Científica Sena Aires. 10(2), 388-399. http://revistafacesa.senaaires.com.br/index.php/revisa/article/view/734/649.

Oliveira, E. H., Silva, C. de M. S. A. da, Verde, R. M. C. L., Soares, L. F., \& Sousa, F. das C. A. (2020). Diabetes mellitus: evaluation and control through laboratory tests in a private service from Teresina, Piauí, Brazil. Research, Society and Development, 9(2), e58922063. https://doi.org/10.33448/rsd-v9i2.2063 Pereira A. S. et al. (2018). Metodologia da pesquisa científica. [free e-book]. Santa Maria/RS. Ed. UAB/NTE/UFSM.

Rodrigues, F. F. A., Brito, L. R. \& Alvim. H. C. O. (2020). Relação do diabetes mellitus tipo II com a depressão e o tratamento com antidepressivos. Revista JRS de estudos acadêmicos. 3(7), 450-462. https://doi.org/10.5281/zenodo.4275851.

SBD. Sociedade Brasileira de Diabetes. (2014). Aplicação de insulina: dispositivos e técnica de aplicação. Diretrizes $2014-2015$. https://www.diabetes.org.br/profissionais/images/pdf/diabetes-tipo-1/002-Diretrizes-SBD-Aplicacao-Insulina-pg219.pdf.

SBD. Sociedade Brasileira de Diabetes. (2017). Recomendações sobre o tratamento injetável do diabetes: insulinas e incretinas. Posicionamento Oficial SBD no 01/2017. https://www.diabetes.org.br/profissionais/images/2017/posicionamento-oficial-sbd-01-2017.pdf.

SBD. Sociedade Brasileira de Diabetes. (2019). Conduta terapêutica no diabetes tipo 2: Algoritmo SBD 2019. Posicionamento Oficial SBD no 01/2019 https://www.diabetes.org.br/publico/images/pdf/sbd_dm2_2019_2.pdf.

SBD. Sociedade Brasileira de Diabetes. (2019). Diretrizes 2019-2020. https://www.diabetes.org.br/profissionais/images/DIRETRIZES-COMPLETA-20192020.pdf.

SBD. Sociedade Brasileira de Diabetes. (2020). Conduta terapêutica em pessoas com diabetes e hipertensão arterial. Posicionamento oficial SBD 03/2020. https://profissional.diabetes.org.br/wp-content/uploads/2021/06/CONDUTA-TERAPEUTICA-HIPERTENSAO-ARTERIAL.pdf.

SBD. Sociedade Brasileira de Diabetes. (2020). Hemoglobina Glicada e Eventos Cardiovasculares em Diabetes Mellitus tipo 2. Diretrizes 2020. https://www.diabetes.org.br/publico/colunas/24-dr-antonio-carlos/63-hemoglobina-glicada-e-eventos-cardiovasculares-em-diabetes-mellitus-tipo-2. 
Research, Society and Development, v. 10, n. 14, e04101421683, 2021

(CC BY 4.0) | ISSN 2525-3409 | DOI: http://dx.doi.org/10.33448/rsd-v10i14.21683

Schonrock, G., Costa, L., Bender, S., \& Linartevichi, V. (2021). Adesão ao tratamento medicamentoso de pacientes idosos hipertensos em uma unidade de saúde da família em Cascavel Paraná. Fag Journal of Health, 3(1), 29-33. https://doi.org/10.35984/fjh.v3i1.298

Scheffel, S. R., Bortolanza. D., Weber. C. S., Costa. L. A., Canani. L H., Santos. K. G., Crispim. D., Roisenberg. I., Lisboa. H. R. K., Tres. G. S., Tschiedel. B. \& Gross. J. L. (2004). Prevalência de complicações micro e macrovasculares e de seus fatores de risco em pacientes com diabetes melito do tipo 2 em atendimento ambulatorial. Revista da associação médica brasileira. 50(3), 263-267. https://doi.org/10.1590/S0104-42302004000300031.

Soares. R. A., \& Romanichen. F. M. D. F. (2020). Fatores relacionados a adesão ao uso de Insulinas em pacientes atendidos na Atenção Básica, Brazilian Journal of Health Review. 3(5), 1517- 15172. https://doi.org/10.34119/bjhrv3n5-297. 